\title{
Overall survival is not the only endpoint to measure clinical relevance
}

\author{
Picardi $\mathrm{C}^{1}$, Di Maio $\mathrm{M}^{2}$ and Addeo $\mathrm{A}^{3 *}$ \\ ${ }^{1}$ Radiation Oncology department, University Hospital of Geneva-HUG, Switzerland \\ ${ }^{2}$ Oncology department, University of Turin, Division of medical oncology, Ordine Mauriziano Hospital, Turin, Italy \\ ${ }^{3}$ Oncology department. University Hospital of Geneva-HUG, Switzerland
}

\section{Commentary}

Small-cell lung cancer (SCLC) represents nearly $13 \%$ of all newly diagnosed lung cancers. Most patients present with extensive disease (ED), and without treatment, the median survival is typically short, ranging from 2 to 4 months [1].

The occurrence of brain metastases (BM) can be associated with severe symptoms and significant impairment in health-related quality of life. At diagnosis, at least $18 \%$ of SCLC patients have already BM, and the incidence of such metastases increases considerably during the course of the disease, reaching $80 \%$ in patients surviving after 2 years [2]. To prevent the disabling clinical consequences of the occurrence of symptomatic BM, one landmark achievement was the adoption of prophylactic cranial irradiation (PCI). This treatment was firstly introduced more than 40 years ago and, since the publication of a meta-analysis in 1999, it has been recommended as standard of care in SCLC patients responding to initial systemic treatment [3]. To date, given the absence of significant improvement in systemic options, PCI still remains the only clinical option to prevent the occurrence of BM. In detail, the meta-analysis was based on 987 patients: the use of PCI confirmed an absolute increase in the 3-year OS rate of 5.4\%, along with a significant decrease of brain metastases incidence (from $58.6 \%$ to $33.3 \%$ after 3 years). Although patients with ED were only $15 \%$, while most patients had ES, the observed benefit was similar in the two subgroups.

Subsequently, the Organisation for Research and Treatment of Cancer (EORTC) conducted a multicenter randomised trial of PCI in SCLC-ED patients who had obtained any response to chemotherapy [3]. Primary endpoint of the trial was the time to development of symptomatic brain metastases, while secondary endpoints were survival, quality of life, toxic effects, and treatment costs. Cumulative risk of developing symptomatic brain metastases within 1 year was 14.6 $\%$ in the PCI group, compared to $40.4 \%$ in the control arm. Notably, although OS was only a secondary endpoint of the study, patients in the PCI group also showed longer OS, with a median survival of 6.7 months compared to 5.4 months $(\mathrm{p}=0.003)$. This study was planned and conducted with a "pragmatic" approach, and brain imaging was not mandatory before enrolment and not routinely performed during follow-up (unless symptoms suggestive of brain metastases). This represents a limitation, implying that a substantial number of patients could have had already asymptomatic brain metastases at the time of randomisation, and in these cases PCI could have been actually a treatment of existing disease, more than a prophylaxis.
Despite this limitation, considering the improvement in OS, PCI strengthened its role in the SCLC ED patients treatment, and an analysis of patterns of care in the USA showed high adherence to guidelines; namely, $98 \%$ of radiation oncologists recommended PCI for patients with extensive-stage SCLC.

More recently, another trial aiming to better define the role of PCI in ED-SCLC was conducted in Japan and get recently published [4]. In detail, in the phase III trial conducted by Takahashi et al. patients were randomised to PCI or monitoring. Unlike the EORTC trial, all patients had brain MRI before the enrolment, to exclude BM and in both arms the MRI was performed every 3 months for the first year, and every 6 months thereafter. The primary endpoint was OS, while time to brain metastases was among secondary endpoints.

This study did not demonstrate any difference in OS between patients receiving PCI (median 11.6 months) and patients assigned to the observation arm (median 13.7 months) (Hazard Ratio 1.27; 95\%CI,0.96-1.68, $\mathrm{p}=0.94$ ). Based on these results, authors concluded that "cranial irradiation is not essential for patients with extensive small cell lung cancer" if regularly assessed by MRI during follow up and treated for symptomatic metastases.

Does this disappointing result in terms of life expectancy represent the end of PCI for patients with ED SCLC? Before a definitive judgement about the role of PCI in this setting, some aspects should be fully highlighted, and a clear understanding of the possible benefits of PCI, beyond the OS prolongation, should be considered. Although different in the OS results, both trials showed that PCI significantly reduces (2- to 3 - fold) the incidence of brain metastases. It is known that a high proportion of SCLC patients will develop brain metastases at some point during the course of their disease and brain lesions can seriously worsen patients' quality of life (QoL). Accordingly, both trials showed a high proportion of patients in the standard arm who required subsequently brain irradiation for new brain lesions. In the study by Takahashi and colleagues, $83 \%$ of patients in the observation arm (compared to $46 \%$ in the PCI arm) needed radiotherapy, and cranial irradiation was offered as well in 59\% of patient the EORTC trial for symptomatic lesions vs $8.3 \%$ in the PCI group. Thus, without PCI, more than half the patients will develop brain metastases, with a clear impact on patients' QOL [5].

*Correspondence to: Alfredo Addeo, Oncology departemtn, rue pierret gentil 4 1205 Geneva, Switzerland, Tel: 0044779930778, E-mail: alfredo.addeo@hcuge.ch

Received: June 13, 2018; Accepted: June 26, 2018; Published: June 29, 2018 
Of course, among oncologists there has always been a common and recurrent concern regarding the possible decline of the neurocognitive functions (NCF) in patients treated by PCI. However, there are actually no data to demonstrate any difference in NCF with or without PCI. Tallet et al. [6] reviewed studies that evaluated NCF after PCI in patients or after WBRT for brain metastases. Three studies looked at NCF after PCI without brain metastases and found no persistent neurocognitive deterioration after PCI [7]. Mini mental state examination was the only parameter assessed within the Japanese trial, and it did not show any difference after 12 and 24 months. Furthermore, most patients already have abnormal neuropsychological testing after chemotherapy and before PCI stressing the importance to assess the chemotherapy impact on NCF. Quality of life was tested in the EORTC trial and the impact of irradiation on functioning scales was moderate. On the other hand, a possible impact on NCF has been described in literature with increased radiation dose, thus delivering radiotherapy for the treatment of brain lesions, where higher doses are needed, could be potentially more toxic.

If from one hand PCI does not seem to necessarily improve OS in SCLC ED, on the other it has a clear role in reducing the incidence of developing brain metastases avoiding a debilitating complication which has a detrimental impact on patients QoL without any derangement on cognitive functions.

Notably the guidelines by the European Society of Medical Oncology, published in 2013, underlined that PCI could be associated with adverse effects such as fatigue and hair loss and with a negative impact on health-related quality of life [8]. According to the guidelines, patients with any response to first-line treatment and who have a reasonably good PS should be evaluated for PCI, but with recommendation B (that means "Strong or moderate evidence for efficacy but with a limited clinical benefit, generally recommended").
Our concern is that, as a result of Takahashi et colleague paper4, the clinical benefit of PCI might be even further undermined, whereas we would strongly encourage oncologists/radiation oncologists to discuss openly the pros and cons of PCI and share the choice with the patients.

In conclusion we agree that OS is undoubtedly an important endpoint but it is not the only one that really matters to us and even more to the patients.

\section{References}

1. Kalemkerian GP (2013) Small cell lung cancer. J Natl Compr Canc Netw 11: 78-98 [Crossref]

2. Seute T, Leffers P, Wilmink JT, ten Velde GPM, Twijnstra A (2006) Response of asymptomatic brain metastases from small-cell lung cancer to systemic first-line chemotherapy. J Clin Oncol 24: 2079-2083.

3. Aupérin A, Arriagada R, Pignon JP, Le Péchoux C, Gregor A, et al. (1999) Prophylactic cranial irradiation for patients with small-cell lung cancer in complete remission. Prophylactic Cranial Irradiation Overview Collaborative Group. $N$ Engl J Med 341: 476-484.

4. Takahashi T,Yamanaka T, Seto T, Harada H, Nokihara H, et al. (2017) Prophylactic cranial irradiation versus observation in patients with extensive-disease small-cell lung cancer: a multicentre, randomised, open-label, phase 3 trial. Lancet Oncol 18: 663-671. [Crossref]

5. Noh T, Walbert $\mathrm{T}$ (2018) Brain metastasis: clinical manifestations, symptom management, and palliative care. Handb Clin Neurol 149: 75-88. [Crossref]

6. Tallet AV, Azria D, Barlesi F, Spano JP, Carpentier AF, et al. (2012) Neurocognitive function impairment after whole brain radiotherapy for brain metastases: actual assessment. Radiat Oncol 7: 77. [Crossref]

7. Grosshans DR, Meyers CA, Allen PK, Davenport SD, Komaki R (2008) Neurocognitive function in patients with small cell lung cancer : effect of prophylactic cranial irradiation. Cancer 112: 589-595. [Crossref]

8. Früh M, De Ruysscher D, Popat S, Crinò L, Peters S, et al. (2013) Small-cell lung cancer (SCLC): ESMO Clinical Practice Guidelines for diagnosis, treatment and follow-up. Ann Oncol 6: 99-105. [Crossref]

Copyright: (C2018 Picardi C. This is an open-access article distributed under the terms of the Creative Commons Attribution License, which permits unrestricted use, distribution, and reproduction in any medium, provided the original author and source are credited. 